\title{
Scaling Behavior of Threshold Epidemics
}

\author{
E. Ben-Naim ${ }^{1}$ and P. L. Krapivsky ${ }^{2}$ \\ ${ }^{1}$ Theoretical Division and Center for Nonlinear Studies, \\ Los Alamos National Laboratory, Los Alamos, NM 87545, USA \\ ${ }^{2}$ Department of Physics, Boston University, Boston, MA 02215, USA
}

\begin{abstract}
We study the classic Susceptible-Infected-Recovered (SIR) model for the spread of an infectious disease. In this stochastic process, there are two competing mechanism: infection and recovery. Susceptible individuals may contract the disease from infected individuals, while infected ones recover from the disease at a constant rate and are never infected again. Our focus is the behavior at the epidemic threshold where the rates of the infection and recovery processes balance. In the infinite population limit, we establish analytically scaling rules for the time-dependent distribution functions that characterize the sizes of the infected and the recovered sub-populations. Using heuristic arguments, we also obtain scaling laws for the size and duration of the epidemic outbreaks as a function of the total population. We perform numerical simulations to verify the scaling predictions and discuss the consequences of these scaling laws for near-threshold epidemic outbreaks.
\end{abstract}

PACS numbers: 02.50.-r, 05.40.-a, 87.23.Cc, 87.10.Mn

\section{INTRODUCTION}

The study of epidemics has a long and fascinating history that dates back to Daniel Bernoulli who modeled the spread of smallpox [1 3$]$. Theoretical models have been quite successful in describing the spread of infectious diseases. Closely related models have been applied to a truly remarkable set of contagious processes including HIV [4, 5], computer viruses [6], spread of technological innovations [7, 8], outbreaks of social and political unrest [9, 10], and rumor propagation [11].

The convenient deterministic framework is most commonly used to model the spread of an epidemic. In this formulation, coupled ordinary differential equations describe the evolution of macroscopic properties such as the average number of infected, the average number of recovered, and so on [12]. However, the deterministic approach cannot capture fluctuations which are inevitable due to the finite size of the population. In many scenarios such as the spread of an infection in an isolated boarding school with say, a hundred children, statistical fluctuations are certainly significant. The stochastic framework which involves evolution equations for the entire probability distribution is instead required to describe finite populations [13 18]. Theoretical analysis of the stochastic framework is challenging, even for the most basic infection processes [19 24], and many questions concerning finite-size effects and extremal properties of the probability distribution functions remain unanswered.

In this paper, we investigate the stochastic version of the classic Susceptible-Infected-Recovered (SIR) infection process. In this model, the population consists of susceptible, infected, and recovered individuals, and the infection spreads through contact between infected and susceptible members of the community [12, 15, 25, 26]. Each infected individual spreads the infection at a certain rate, denoted by $\alpha$, while infected individuals recover at a rate set to one. The epidemic threshold is $\alpha=1$. Below the threshold, the infection cannot maintain itself; above the threshold, the infection can take off.

We focus on epidemic outbreaks at or near the threshold [26 32]. Such "threshold epidemics" are especially interesting. From a theoretical viewpoint, the infection and the recovery processes are in some sense in perfect balance precisely at the threshold. While most infections are small, large outbreaks may occasionally happen, and hence, threshold epidemics exhibit large fluctuations. From a practical viewpoint, human efforts at disease prevention reduce the infection rate thereby driving infectious diseases from the pandemic to the endemic phase 25]. Meanwhile, natural evolution may increase the infection rate of diseases hovering just below the threshold, thereby enhancing the likelihood of an outbreak [33]. These epidemics are subtle: they may be difficult to detect as well as difficult to control.

We first consider infinite populations and focus on the behavior precisely at the epidemic threshold. We analyze the rate equation for the probability $P_{i, r}(t)$ that the number of infected equals $i$ and the number of recovered equals $r$ at time $t$. The typical number of infected grows linearly with time while the typical number of recovered grows quadratically with time. We show that the joint distribution function obeys the scaling form

$$
P_{i, r}(t) \simeq t^{-4} \Phi\left(i t^{-1}, r t^{-2}\right)
$$

We obtain the Laplace transform of the scaling function $\Phi(\xi, \eta)$ analytically, and present a comprehensive asymptotic analysis. We also discuss scaling properties of the respective single-population distribution functions for the number of infected or the number of removed.

Next, we consider finite populations. We combine the infinite population results with heuristic arguments to derive scaling laws for the size and duration of outbreaks in a finite population of size $N$. At or near the epidemic threshold, the effective infection rate is reduced because the population is finite. We find that the maximal size of the outbreak, $M$, and the maximal duration of the 
outbreak, $T$, obey the nontrivial scaling laws

$$
M \sim N^{2 / 3}, \quad \text { and } T \sim N^{1 / 3} .
$$

We also formulate the range of infection rates near the epidemic threshold for which these scaling laws apply.

The rest of this paper is organized as follows. We begin with the definition of the infection process, given in Section II. In section III, we treat the infinite population limit by using the rate equation approach. First, we derive the outbreak size distribution. We then compute two single-variable distributions - the probability distribution for the number of infected individuals and the probability distribution of the number of recovered individuals. Next, we discuss scaling and extremal properties of the joint distribution function. In section IV, we consider finite populations and obtain finite-size scaling laws for the size and duration of the outbreaks. We discuss the results in section $\mathrm{V}$, and the appendix details some necessary inverse Laplace transforms.

\section{THE INFECTION PROCESS}

The spread of infectious diseases has been widely studied on regular and irregular spatial structures such as lattices [34-42] and complex networks [43-45]. Nevertheless, many characteristics of stochastic epidemics, e.g. finite-size properties, remain open questions even for perfectly mixed populations. Throughout this paper we assume that the populations are well-mixed so that any infected individual can spread the disease to any susceptible individuals.

In the ubiquitous Susceptible-Infected-Recovered (SIR) infection process, the population includes three types of of individuals:

S Uninfected individuals who are susceptible to the infection.

I Infected individuals who are actively spreading the disease.

$\mathbf{R}$ Individuals who are neither infected nor susceptible including those who have been infected and subsequently recovered, or became immune, or removed.

An individual may proceed from type $\mathbf{S}$ to $\mathbf{I}$ to $\mathbf{R}$. In this simplified model, all infected individuals may spread the disease.

We investigate the continuous-time version of SIR infection process [15]. At any given moment, the population consists of $s$ susceptible, $i$ infected, and $r$ recovered individuals; the size of the total population, $N=s+i+r$, remains fixed. The sub-populations change due to two competing processes - infection and recovery. An infected individual may infect a susceptible one with rate $\alpha / N$, where $\alpha$ is the infection rate:

$$
(s, i, r) \stackrel{\alpha s i / N}{\longrightarrow}(s-1, i+1, r) .
$$

The overall infection rate is proportional to the size of the susceptible population times the size of the infected population. Infected individuals recover at a constant rate,

$$
(s, i, r) \stackrel{i}{\longrightarrow}(s, i-1, r+1) .
$$

Without loss of generality, the recovery rate is set to one.

We consider the natural initial condition where a single infected individual is embedded in a "sea" of susceptible individuals, that is, $(s, i, r)=(N-1,1,0)$ at time $t=0$. The infection process ends when no infected individuals remain, $(s, i, r)=(N-n, 0, n)$. The final number infected, $n$, measures the size of the epidemic outbreak.

\section{INFINITE POPULATIONS}

Since the infection process is random, the size of the epidemic outbreak is a stochastic quantity. First, we investigate the distribution of this quantity as its basic characteristics show how the infection process can be in one of two phases: an endemic phase where a microscopic number of individuals are infected and a pandemic phase where a macroscopic number of individuals are infected.

In this section we analyze the infinite population limit, $N \rightarrow \infty$. Let $G_{n}$ be the probability that the size of the epidemic outbreak equals $n$. For the initial condition $(s, i, r)=(N-1,1,0)$, the total infection rate and the total recovery rate are $\alpha$ and 1 as follows from (3) -(4). In the very first step, the infected individual either recovers, or a second individual become infected. The former happens with probability $G_{1}=1 /(1+\alpha)$, while the latter occurs with the complementary probability $\alpha /(1+\alpha)$. Hence, the distribution $G_{n}$ obeys the recursion equation

$$
G_{n}=\frac{\alpha}{1+\alpha} \sum_{i+j=n} G_{i} G_{j}+\frac{1}{1+\alpha} \delta_{n, 1}
$$

valid for all $n \geq 1$. The convolution term corresponds to the case where an additional individual become infected - in this situation there are two infection processes, and in the infinite population limit these two processes are completely independent [46, 47]. Starting with $G_{1}=$ $1 /(1+\alpha)$, the recursion equation gives $G_{2}=\alpha /(1+\alpha)^{3}$, $G_{3}=2 \alpha /(1+\alpha)^{5}$, etc.

We now introduce the generating function

$$
\mathcal{G}(z)=\sum_{n \geq 1} G_{n} z^{n}
$$

Using the generating function we convert the infinite set of recursion equations (5D) into the quadratic equation, $(1+\alpha) \mathcal{G}=\alpha \mathcal{G}^{2}+z$. Out of the two solutions, the proper one satisfies the requirement $\mathcal{G}(z=0)=0$,

$$
\mathcal{G}(z)=\frac{1+\alpha-\sqrt{(1+\alpha)^{2}-4 \alpha z}}{2 \alpha} .
$$


The behavior of $G(z)$ in the limit $z \rightarrow 1$ reveals the basic characteristics of the infection process. The probability $G_{\text {finite }}$ that only a finite number of individuals are infected is given by

$$
G_{\text {finite }}= \begin{cases}1 & \alpha \leq 1 \\ \alpha^{-1} & \alpha>1\end{cases}
$$

The derivation of (8) follows from (77) and $G_{\text {finite }}=\sum_{n>1} G_{n}=\mathcal{G}(1)$. The threshold value $\alpha_{c}=1$ separates two regimes of behavior — an endemic regime and a pandemic regime. Below the threshold, the number of infected individuals is always finite. Above the threshold, there is a finite probability that a finite fraction of individuals becomes infected, and as a consequence, the average size of the epidemic outbreak is macroscopic, that is, proportional to the size of the total population.

Also, the average number of infected individuals readily follows from the generating function, viz. $\langle n\rangle=\sum_{n} n G_{n}=\mathcal{G}^{\prime}(1)$. Equation (7) gives

$$
\langle n\rangle=\frac{1}{1-\alpha}, \quad \alpha<1
$$

As expected, the size of the outbreak diverges in the vicinity of the threshold.

To find $G_{n}$, we expand the generating function $G(z)$ in powers of $z$. The distribution of outbreak size is a product of an exponential and an algebraic factor, expressed as a ratio of Gamma functions,

$$
G_{n}=\frac{1}{(1+\alpha) \sqrt{\pi}} \frac{\Gamma\left(n-\frac{1}{2}\right)}{\Gamma(n+1)}\left[1-\left(\frac{1-\alpha}{1+\alpha}\right)^{2}\right]^{n-1}
$$

By using the asymptotic behavior $\Gamma(x+a) / \Gamma(x) \simeq x^{a}$ as $x \rightarrow \infty$, we conclude that at the epidemic threshold, $\alpha=1$, the size distribution has a power-law tail

$$
G_{n} \simeq(4 \pi)^{-1 / 2} n^{-3 / 2},
$$

as $n \rightarrow \infty$. For threshold epidemics, there is balance between infection and recovery, as indicated by (3)-(4). While the majority of outbreaks are small, the algebraic behavior (10) suggests that there is a considerable likelihood for large outbreaks to occur.

In the remainder of this section, we focus on the behavior at the epidemic threshold, $\alpha=1$. We begin with the probability $P_{i}(t)$ that there are $i$ infected individuals at time $t$. Irrespective of the infection rate, this distribution function satisfies a closed evolution equation, and for the critical $\alpha=1$ case, $P_{i}(t)$ satisfies

$$
\frac{d P_{i}}{d t}=(i-1) P_{i-1}+(i+1) P_{i+1}-2 i P_{i} .
$$

The initial condition is $P_{i}(0)=\delta_{i, 1}$. Equations (11) are closed because in the infinite population limit, $N \rightarrow \infty$, the population of infected individuals does not depend on the populations of susceptible and recovered individuals. We comment that the master equation (11) is a discrete diffusion equation with a diffusion coefficient equal to the size of the infected population.

To solve (11) we use the exponential ansatz: $P_{i}(t)=\Psi(t)[\psi(t)]^{i-1}$ for $i>0$, with the initial conditions $\Psi(0)=1$ and $\psi(0)=0$ to assure the validity of $P_{i}(0)=\delta_{i, 1}$. This ansatz reduces the infinite set of equations (11) to two ordinary differential equations, $d \psi / d t=(1-\psi)^{2}$ and $d \Psi / d t=2(\psi-1) \psi$. Solving these coupled equations we obtain

$$
P_{i}(t)=\frac{1}{(1+t)^{2}}\left(\frac{t}{1+t}\right)^{i-1}
$$

for $i>0$. Thus, the size distribution is purely exponential.

The quantity $P_{0}(t)$ gives the probability that the infection process has subsided by time $t$. From $d P_{0} / d t=P_{1}$ and the initial condition $P_{0}(0)=0$, we obtain $P_{0}(t)=t /(1+t)$. Equivalently, one can deduce this result from the normalization requirement $\sum_{i \geq 0} P_{i}=1$. Note also that the survival probability $P(t)$, defined as the probability that the infection remains active at time $t$, is simply $P(t)=1-P_{0}(t)$, and hence, it is given by

$$
P(t)=\frac{1}{1+t}
$$

In the long time limit, the survival probability decays algebraically, $P \simeq t^{-1}$.

Interestingly, the first moment of the distribution $P_{i}$ is conserved, $\sum_{i} i P_{i}=1$, and in this sense, the competing processes of infection and recovery balance when $\alpha=1$. Hence, if we restrict our attention to active outbreaks, the average number of infected individuals grows linearly with time, $\langle i\rangle=1+t$, where $\langle i\rangle=\sum_{i} i P_{i} / P(t)$.

In the long-time limit, the distribution $P_{i}(t)$ has the scaling form

$$
P_{i}(t) \simeq t^{-2} \Phi\left(i t^{-1}\right), \quad \Phi(\xi)=e^{-\xi} .
$$

This scaling behavior immediately follows from (12) in the limit $i \rightarrow \infty$ and $t \rightarrow \infty$ with the scaling variable $\xi=i / t$ kept fixed.

The master equation (11) is closed since the infected population is decoupled from the other two populations. In contrast, the recovered population is coupled to the infected population. Therefore, we must analyze the joint distribution $P_{i, r}(t)$, that is, the probability there are $i$ infected and $r$ recovered at time $t$. Of course, the joint distribution function gives a complete description of the state of the system and for example, $P_{i}=\sum_{r \geq 0} P_{i, r}$. The joint distribution obeys

$$
\frac{d P_{i, r}}{d t}=(i-1) P_{i-1, r}+(i+1) P_{i+1, r-1}-2 i P_{i, r}
$$

and $P_{i, r}(0)=\delta_{i, 1} \delta_{r, 0}$. In this rate equation, the first gain term accounts for infection, while the second gain term 
represents recovery. For small $i$ and $r$ one can compute the joint distribution recursively: $P_{1,0}=e^{-2 t}, P_{0.1}=$ $\left(1-e^{-2 t}\right) / 2, P_{1,1}=e^{-2 t}\left[2 t-\left(1-e^{-2 t}\right)\right] / 2$, etc. [48].

Generally, we use the generating function

$$
\mathcal{P}(u, v)=\sum_{i \geq 0} \sum_{r \geq 0} P_{i, r} u^{i} v^{r} .
$$

By multiplying (15) by $u^{i} v^{r}$ and summing over all $i$ and $r$, we find that the generating function obeys

$$
\frac{\partial \mathcal{P}}{\partial t}=\left(u^{2}-2 u+v\right) \frac{\partial \mathcal{P}}{\partial u} .
$$

The initial condition is $\mathcal{P}_{0} \equiv \mathcal{P}(u, v)=u$ where $\left.\mathcal{P}_{0} \equiv \mathcal{P}\right|_{t=0}$.

We can transform equation (17) into the wave equation

$$
\frac{\partial \mathcal{P}}{\partial t}=\frac{\partial \mathcal{P}}{\partial w}
$$

To establish this equation, we introduce the variable

$$
\begin{aligned}
w & =\int_{0}^{u} \frac{d u^{\prime}}{\left(u^{\prime}\right)^{2}-2 u^{\prime}+v} \\
& =\frac{1}{2 \sqrt{1-v}} \ln \frac{1+\sqrt{1-v}-u}{1-\sqrt{1-v}-u} \frac{1-\sqrt{1-v}}{1+\sqrt{1-v}}
\end{aligned}
$$

The solution to equation (17) is simply $\mathcal{P}_{0}(w+t)$. Since $\mathcal{P}_{0}=u$, we need to obtain $u$ in terms of the variable $w$ and then, replace $w$ with $w+t$ to obtain the generating function explicitly. From (19), the expression

$$
\begin{aligned}
u & =1-\sqrt{1-v} \\
& -2 \sqrt{1-v}\left[\frac{1+\sqrt{1-v}}{1-\sqrt{1-v}} e^{2 w \sqrt{1-v}}-1\right]^{-1}
\end{aligned}
$$

gives $\mathcal{P}_{0}=u$ in terms of $v$ and $w$. As a function of the variables $w$ and $v$, the generating function becomes

$$
\begin{aligned}
\mathcal{P}(w, v) & =1-\sqrt{1-v} \\
& -2 \sqrt{1-v}\left[\frac{1+\sqrt{1-v}}{1-\sqrt{1-v}} e^{2(w+t) \sqrt{1-v}}-1\right]^{-1} .
\end{aligned}
$$

This result is derived from (20) by replacing $w$ with $w+t$. In terms of the original variables, the generating function is

$$
\mathcal{P}(u, v)=1-\sqrt{1-v}-2 \sqrt{1-v} \frac{1-\sqrt{1-v}-u}{D-(E-1) u} .
$$

We obtained this result by expressing $\exp [2 w \sqrt{1-v}]$ in terms of $u$. In writing (21), we also used the shorthand notations

$$
E=e^{2 t \sqrt{1-v}}, \quad D=(E+1) \sqrt{1-v}+E-1 .
$$

For example, we can verify that the generating function yields the size distribution of outbreaks. In the longtime limit, the last term on the right-hand-side of (21) vanishes since the quantities $D$ and $E$ are both divergent. With the shorthand notation $\mathcal{P}_{\infty} \equiv \lim _{t \rightarrow \infty} \mathcal{P}$, we have $\mathcal{P}_{\infty}(u, v)=1-\sqrt{1-v}$. By substituting $\alpha=1$ into (7), we confirm that $P_{i, r}(t) \rightarrow \delta_{i, 0} G_{r}$ when $t \rightarrow \infty$.

First, we analyze the probability distribution $\Pi_{r}(t)$. By definition, $\Pi_{r}(t)$ is the probability to have $r$ recovered individuals at time $t$. Of course, $\Pi_{r}(t)=\sum_{i \geq 0} P_{i, r}(t)$. The distribution of recovered differs from the distribution of infected in that in the long-time limit, it approaches a nonzero value, $\Pi_{r} \rightarrow G_{r}$, whereas $P_{i} \rightarrow 0$ for all $i>0$. To study the long-time asymptotic behavior, we write $\Pi_{r}(t)=G_{r}+H_{r}(t)$. We have $H_{r}(t) \rightarrow 0$ as $t \rightarrow \infty$. The corresponding generating function $H(v)=\sum_{r \geq 0} H_{r} v^{r}$ is given by

$$
H(v)=\frac{2 \sqrt{1-v}}{e^{2 t \sqrt{1-v}}+1} .
$$

We obtain this expression from the joint generating function (21) by setting $u=1, H(v)=\mathcal{P}(1, v)-P_{\infty}(1, v)$ where $\mathcal{P}_{\infty}(1, v)=1-\sqrt{1-v}$.

According to the scaling behavior (14), the typical size of the infected population grows linearly with time, $i \sim t$. Heuristically, $d r / d t \sim i$ since the recovery rate is constant. Consequently, the typical size of the recovered population is quadratic, $r \sim t^{2}$, and hence, we expect the scaling behavior

$$
H_{r}(t) \simeq t^{-3} \varphi\left(r t^{-2}\right)
$$

The Laplace transform of the scaling function $\varphi(\eta)$ follows immediately from behavior of the generating function (23) in the limit $v \rightarrow 1$. We take the limits $v \rightarrow 1$ and $t \rightarrow \infty$ while keeping the variable $b=t^{2}(1-v)$ fixed. In this limit, we have $v^{r} \rightarrow e^{-b \eta}$ and the sum over $r$ turns into the integral

$$
\sum_{r \geq 0} v^{r} \rightarrow t^{2} \int_{0}^{\infty} d \eta e^{-b \eta}
$$

By using this scaling transformation along with Eq. (23), we find the Laplace transform of the scaling function

$$
\int_{0}^{\infty} d \eta e^{-b \eta} \varphi(\eta)=\frac{2 \sqrt{b}}{e^{2 \sqrt{b}}+1} .
$$

The inversion of this Laplace transform through integration in the complex plane is detailed in the Appendix where we show that the scaling function $\varphi(\eta)$ is given by

$$
\varphi(\eta)=2 \pi^{2} \sum_{k=0}^{\infty}\left(k+\frac{1}{2}\right)^{2} e^{-\pi^{2}\left(k+\frac{1}{2}\right)^{2} \eta}-\frac{1}{\sqrt{4 \pi \eta^{3}}} .
$$

We now substitute this expression into the scaling form (24) and observe that the algebraic factor $(4 \pi)^{-1 / 2} \eta^{-3 / 2}$ and the final distribution $G_{r}$ cancel each other. Thus, the distribution $\Pi_{r}(t)$ has the same scaling form as the distribution $H_{r}(t)$,

$$
\Pi_{r}(t) \simeq t^{-3} \phi\left(r t^{-2}\right) .
$$


The scaling function $\phi(\eta)$ is given by the sum

$$
\phi(\eta)=2 \pi^{2} \sum_{k=0}^{\infty}\left(k+\frac{1}{2}\right)^{2} e^{-\pi^{2}\left(k+\frac{1}{2}\right)^{2} \eta} .
$$

The leading asymptotic behaviors of the scaling function $\phi(\eta)$ are

$$
\phi(\eta) \simeq \begin{cases}(4 \pi)^{-1 / 2} \eta^{-3 / 2} & \eta \rightarrow 0 \\ \left(\pi^{2} / 2\right) e^{-\pi^{2} \eta / 4} & \eta \rightarrow \infty\end{cases}
$$

The algebraic behavior in the small- $\eta$ limit is consistent with the power-law tail (10). To obtain this behavior, we simply convert the sum in (28) into an integral. We note that with the scaling form (27) and the small- $\eta$ divergence, the quantity $\sum_{r} \Pi_{r}$ is indeed finite. Also, the first term in the series yields the exponential behavior in the large- $\eta$ limit. Hence, both the distribution of recovered and the distribution of infected have exponential tails.

We now analyze the joint distribution $P_{i, r}(t)$. We restrict our attention to active epidemics that correspond to $i>0$. Thus, we focus on the following component of the joint generating function

$$
\mathcal{F}(u, v)=\sum_{i \geq 1} \sum_{r \geq 0} P_{i, r} u^{i} v^{r}
$$

This component follows directly from the generating function, $\mathcal{F}(u, v)=\mathcal{P}(u, v)-\mathcal{P}(0, v)$, and by using the explicit solution (21), we obtain

$$
\mathcal{F}(u, v)=\frac{4 E u(1-v)}{D[D-(E-1) u]} .
$$

The scaling behaviors (14) and (27) for the singlepopulation distributions imply that the joint distribution function adheres to the scaling form (1) stated in the introduction. Since the survival probability $P(t)=$ $\sum_{i \geq 1} \sum_{r} P_{i, r}(t)$ decays with time according to (13), the scaling function is normalized $\iint d \eta d \xi \Phi(\xi, \eta)=1$.

The above analysis suggests use of the joint Laplace transform

$$
f(a, b)=\int_{0}^{\infty} \int_{0}^{\infty} d \xi d \eta e^{-a \xi-b \eta} \Phi(\xi, \eta),
$$

that is merely the continuous counterpart of the generating function. To obtain $f(a, b)$ from $\mathcal{F}(u, v)$ given in (31), we take the limits $t \rightarrow \infty, u \rightarrow 1$, and $v \rightarrow 1$ while keeping the variables

$$
a=(1-u) t \quad \text { and } \quad b=(1-v) t^{2}
$$

fixed. By taking these limits, we observe that the righthand side of (30) becomes $t^{-1} f(a, b)$, and find the joint Laplace transform in explicit form

$$
f(a, b)=\left(\frac{\sqrt{b}}{\sinh \sqrt{b}}\right)^{2} \frac{1}{a+\sqrt{b} \operatorname{coth} \sqrt{b}} .
$$

We stress that the quantity $f(a, b)$ describes only active infection processes. One can verify that $f(0,0)=1$. Moreover, in the $b \rightarrow 0$ limit, we have $f(a, 0)=1 /(1+a)$, for which the inverse Laplace transform is immediate, $\int d \eta \Phi(\xi, \eta)=e^{-\xi}$. Indeed, we recover the scaling function $\Phi(\xi)$ in Eq. (14).

Since the inverse Laplace transform of $f(a, b)$ with respect to the variable $b$ is immediate, we have

$$
\int d \eta e^{-b \eta} \Phi(\xi, \eta)=\left(\frac{\sqrt{b}}{\sinh \sqrt{b}}\right)^{2} e^{-\xi \sqrt{b} \operatorname{coth} \sqrt{b}} .
$$

The Appendix outlines how to invert this Laplace transform to obtain the leading asymptotic behaviors for large$\eta$ and small- $\eta$,

$$
F(\xi, \eta) \simeq \begin{cases}\frac{4(1+\xi / 2)^{3}}{\pi^{1 / 2} \eta^{7 / 2}} \exp \left[-\frac{(1+\xi / 2)^{2}}{\eta}\right] & \eta \rightarrow 0, \\ \frac{\pi^{2} 2^{1 / 4}}{\xi^{3 / 4}} \exp \left[-\pi^{2} \eta+\pi \sqrt{8 \xi \eta}-\xi\right] & \eta \rightarrow \infty .\end{cases}
$$

These limiting behaviors apply for a fixed value of $\xi$.

The function $f(a, b)$ captures all moments of the scaling function $\Phi(\xi, \eta)$. For example, by expanding equation (33) as a Taylor series in the conjugate variables $a$ and $b, f(a, b)=1-a-\frac{2}{3} b+a b+\cdots$, and comparing with the definition (32), we obtain the lowest-order moments $\langle\xi\rangle=1,\langle\eta\rangle=\frac{2}{3}$, and $\langle\xi \eta\rangle=1$. In particular,

$$
\langle\xi \eta\rangle>\langle\xi\rangle\langle\eta\rangle
$$

so there is positive correlation between the size of the two populations. Intuitively, we expect that long-lasting epidemic outbreaks involve large numbers of infected and recovered, while the opposite is true for short-lived outbreaks.

For completeness, we mention that the above analysis can be repeated for inactive outbreaks. Starting with $\mathcal{P}(0, v)$ given in (21) and following the steps leading to (27), we find that the distribution $P_{0, r}(t)$ that an epidemic outbreak has ended by time $t$ and that the size of the outbreak equals $r$, has the scaling form $P_{0, r}(t) \simeq t^{-3} \tilde{\phi}\left(r t^{-2}\right)$. The scaling function $\tilde{\phi}(\eta)$ resembles $\phi(\eta)$ given in (28)

$$
\tilde{\phi}(\eta)=2 \pi^{2} \sum_{k=1}^{\infty} k^{2} e^{-\pi^{2} k^{2} \eta}
$$

The leading asymptotic behavior in the small- $\eta$ limit is identical to that in (29), and again, there is exponential decay, albeit with twice the coefficient, $\tilde{\phi}(\eta) \simeq 2 \pi^{2} \exp \left(-\pi^{2} \eta\right)$ in the large- $\eta$ limit. As expected, inactive outbreaks dominate the size distribution of the recovered population at large times.

\section{FINITE POPULATIONS}

The zeroth and first-order moments of the distribution $G_{r}$ given in equations (8) and (9) show that the size of 


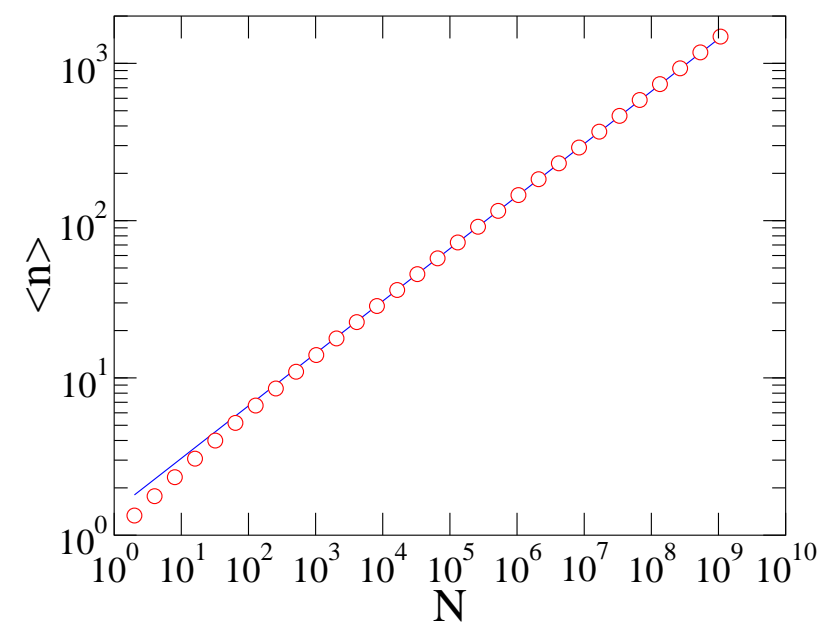

FIG. 1: The average outbreak size versus the population size for the SIR infection process at the epidemic threshold $(\alpha=1)$. Shown are Monte Carlo simulation results representing an average over $10^{9}$ independent realizations of the infection process (circles). A line of slope $1 / 3$ is also shown as a reference.

the epidemic outbreak is microscopic below the epidemic threshold, $\alpha<1$, but macroscopic above the threshold, $\alpha>1$. These results hold for infinite populations, yet real-world epidemic outbreaks involve finite populations, and there is practical need for understanding how basic characteristics such as the average size and the average duration of the epidemic depend on the size of the population, $N$.

Finite-size effects are most pronounced in the vicinity of the epidemic threshold. Let us consider large but finite populations, $N \gg 1$, and let us consider a scenario in which the susceptible population has been depleted by $n$, that is, $s=N-n$. From the very definition of the infection process (3), we determine that the infection rate is reduced, $\alpha \rightarrow \alpha_{*}(N)$, with

$$
\alpha_{*}(N)=\alpha\left(1-\frac{n}{N}\right),
$$

because the total population is finite. Clearly, as the large "reservoir" of susceptible individuals shrinks, the infection process weakens.

Let us now consider the average size of the outbreak for a threshold epidemic $(\alpha=1)$. We assume that there is a maximal outbreak size $M$, and that the outbreak can not exceed this size due to depletion in the number of susceptible individuals. On the one hand, equations (38) and (91) suggest that $\langle n\rangle \sim N / M$. On the other hand, the algebraic distribution (10) gives a second estimate $\langle n\rangle \sim \sum_{n \leq M} n^{-1 / 2} \sim M^{1 / 2}$ for the average size of the outbreak. Equating these two estimates, $N / M \sim M^{1 / 2}$, we conclude that $M \sim N^{2 / 3}$ as stated in (2). While a naive interpretation of the effective infection rate (38) would suggest that the cutoff is proportional to the total population, we find that threshold epidemics have a substantially smaller upper bound.

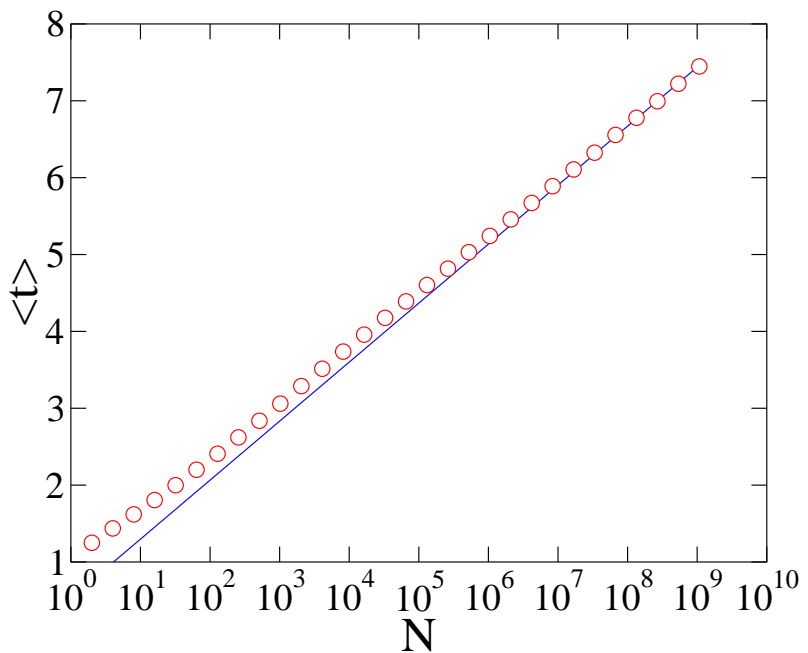

FIG. 2: The average outbreak duration at the epidemic threshold versus the population size. Simulation results, obtained from an average over $10^{9}$ realizations (circles) are compared with the reference line $(1 / 3) \ln N+C$.

Using $\langle n\rangle \sim N / M$ and $M \sim N^{2 / 3}$ we see that the average size of a threshold epidemic grows as

$$
\langle n\rangle \sim N^{1 / 3} .
$$

This interesting scaling law was established by MartinLöf [19] and it has been confirmed in several recent studies 20 24]. Figure 1] shows a numerical verification of this behavior. For finite populations, this scaling law holds in the neighborhood (often termed critical or scaling windows) of the epidemic threshold. To estimate the size of this neighborhood, we simply compare (39) with (91). The size of the outbreak grows sub-linearly in the population size as long as

$$
|1-\alpha| \sim N^{-1 / 3} .
$$

Of course, this neighborhood shrinks as the size of the population grows. Yet, for moderate populations, the size of this neighborhood is considerable.

The scaling law (39) indicates that for finite populations, there are actually three regimes of behavior. Well below the epidemic threshold, a finite number of individuals becomes infected. Well above the threshold, a finite fraction of the population becomes infected. In the vicinity of the threshold, the size of the outbreak grows as the $1 / 3$ power of the population size,

$$
\langle n\rangle \sim \begin{cases}\mathcal{O}(1) & \zeta \rightarrow \infty \\ N^{1 / 3} Y(\zeta) & \zeta=\mathcal{O}(1), \\ N & \zeta \rightarrow-\infty\end{cases}
$$

with the scaling variable $\zeta=N^{1 / 3}(1-\alpha)$. A finite value of $\zeta$ indicates that the infection rate is in the neighborhood of the threshold. (The scaling function $Y(\zeta)$ can be extracted from Refs. [19, 21 23].) 


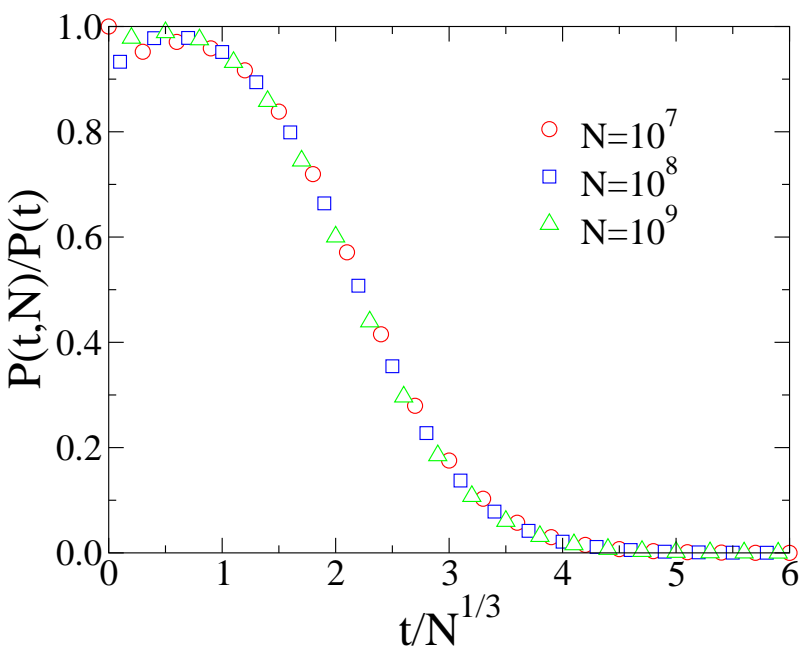

FIG. 3: The survival probability at the epidemic threshold. Shown is the normalized survival probability $P(t, N) / P(t)$ versus the normalized duration time $t / N^{1 / 3}$. The data corresponds to an average over $10^{8}$ realizations.

The duration of a threshold epidemic also adheres to a finite-size scaling law. Since the size of the recovered population grows quadratically with time, $r \sim t^{2}$, as implied for example by the scaling behavior (14), we conclude that there is a maximal time scale $T$, and that the duration of a near-threshold outbreak may not exceed this scale. We can estimate the time scale $T \sim N^{1 / 3}$ stated in (2), from the heuristic argument $T^{2} \sim M$. The average duration of the infection process follows from the survival probability, $\langle t\rangle=-\int_{0}^{T} d t t d P / d t$, and using $P \sim t^{-1}$, we find that the average duration grows logarithmically with time (Fig. 2),

$$
\langle t\rangle \simeq \frac{1}{3} \ln N
$$

Logarithmic growth, albeit with a unit prefactor, was predicted by Ridler-Rowe [49].

The scales $M \sim N^{2 / 3}$ and $T \sim N^{1 / 3}$ fully characterize the size and duration of the infection process when $\alpha=1$. For example, the survival probability $P(t, N)$ to have at least one infected at time $t$ obeys $P(t, N) / P(t) \rightarrow$ $\mathcal{S}\left(t / N^{1 / 3}\right)$ (Fig. 3). However, the simulations show that the convergence to this scaling form is not uniform - it is slow for short durations but fast at large durations.

We performed numerical simulations to check the scaling predictions for the case $\alpha=1$. Our numerical method is merely a Monte Carlo procedure to solve the master equation (15). We conveniently keep track of two populations, $s$ and $i$, from which the overall infection rate and recovery rate are respectively $R_{i}=s i / N$ and $R_{r}=i$. The probabilities $P_{i}=R_{i} /\left(R_{i}+R_{r}\right)$ and $P_{r}=R_{r} /\left(R_{i}+R_{r}\right)$ that the following step involves infection or recovery are calculated and then, the populations are updated accordingly,

$$
(s, i) \rightarrow \begin{cases}(s-1, i+1) & \text { with probability } P_{i} \\ (s, i-1) & \text { with probability } P_{r}\end{cases}
$$

The infection process ends when $i=0$ for the first time. Time is updated by the inverse of the total rate $t \rightarrow t+1 /\left(R_{i}+R_{r}\right)$. With this straightforward numerical procedure, we can simulate as many as $10^{9}$ independent runs in populations as large as $N=10^{9}$. As shown in figures 1 and 2, there is excellent agreement between the numerical results and the theoretical predictions for the average size (39) and the average duration (42).

\section{DISCUSSION}

We investigated various statistical properties of the SIR infection process at a threshold infection rate. We analyzed the rate equations for the two-population distribution function that characterizes the probability that the system has a specified number of infected and recovered individuals. Our analysis yields scaling behavior in the asymptotic long-time limit and gives extremal properties of the joint distribution function as well as the associated single-population distributions. We used these infinite population results to justify scaling laws for finite populations.

Outbreaks in the vicinity of the epidemic threshold have a distinct size, characterized by non-trivial powerlaw dependence on the total population size. This scaling behavior applies in the vicinity of the epidemic threshold. The size of this neighborhood, $N^{-1 / 3}$, is larger than the canonical value $N^{-1 / 2}$ expected from the traditional large-size expansions or from the deterministic description [50]. Therefore, statistical fluctuations and finite population effects are pronounced and may be subtle near the epidemic threshold. We note the an identical scaling arises near the percolation point for Erdős-Rényi random graphs [51, 52]. Additional connections between SIR infection processes and random graphs were reported recently [23].

The scaling laws for the time dependence and the size dependence are useful. For example, the scaling laws for the critical kinetics can be used to find the epidemic threshold in numerical simulations of infection processes on complex networks for which the threshold is not known analytically. Furthermore, the number of coupled ordinary equations needed to compute the joint distribution $P_{i, r}$ numerically is in principle quadratic with $N$. However, the scaling laws $i \sim N^{1 / 3}$ and $r \sim N^{2 / 3}$ imply that the relevant number of equations is much smaller, being proportional to $N$.

For large but finite populations, we understand the basic scaling laws, but much less is known about finite-size scaling functions. The only exception is the known scaling function that gives the size distribution of the outbreaks at the epidemic threshold [19, 21]. The analytic 
determination of the scaling function characterizing the duration of outbreaks near the epidemic threshold is a challenging problem because there is no closed equation for the total duration of the outbreak [18].

Finally, we mention that in this study we assumed that all individuals can interact. In most applications, the spatial [34 42] or network [43 45] structure of the infected domain play an important role. Finding the corresponding scaling functions for the time-dependent behavior at the critical point or for the finite-size scaling are also challenging open problems.

\section{Acknowledgments}

We are grateful to Aric Hagberg for initial collaboration and thank Tibor Antal and David Kessler for useful correspondence. We also acknowledge support for this research by DOE grant DE-AC52-06NA25396.
[1] M. McNeill, Plagues and People (Anchor Books, New York, 1989).

[2] M. Oldstone, Viruses, Plagues, and History (Oxford University Press, Oxford, 1998).

[3] D. Bernoulli, Mem. Math. Phys. Acad. Roy. Sci., Paris, 1-45 (1760).

[4] A. S. Perelson and P. W. Nelson, SIAM Rev. 41, 3 (1999).

[5] M. A. Nowak and R. May, Virus Dynamics: Mathematical Principles of Immunology and Virology (Oxford University Press, Oxford, 2001).

[6] M. A. Ludwig, The Giant Black Book of Computer Viruses (American Eagle Publications Inc., Show Low, 1998).

[7] E. M. Rogers, Diffusion of Innovations (Free Press, New York, 2003).

[8] P. L. Krapivsky, S. Redner, and D. Volovik, J. Stat. Mech. P12003 (2011).

[9] M. Granovetter, Am. J. Sociol. 83, 1420 (1978).

[10] S. Lohmann, World Politics 47, 42 (1994).

[11] D. J. Day and D. G. Kendall, Nature 204, 496 (1964).

[12] J. D. Murray, Mathematical Biology. I. An Introduction (Springer-Verlag, New York, 2002).

[13] A. G. McKendrick, Proc. Edin. Math. Soc. 14, 98 (1926).

[14] N. T. J. Bailey, Biometrika 37, 193-202 (1950); ibid 40, 177-185 (1953).

[15] N. T. J. Bailey, The Mathematical Theory of Infectious Diseases (Oxford University Press, Oxford, 1987).

[16] D. P. Maki and M. Thompson, Mathematical Models and Applications, with emphasis on the social, life, and management sciences (Englewood Cliffs, N.J., Prentice-Hall, 1973).

[17] H. Andersson and T. Britton, Stochastic Epidemic Models and Their Statistical Analysis (New York, Springer, 2000).

[18] P. L. Krapivsky, S. Redner and E. Ben-Naim, A Kinetic View of Statistical Physics (Cambridge: Cambridge University Press, 2010).

[19] A. Martin-Löf, J. Appl. Probab. 35, 671 (1998).

[20] E. Ben-Naim and P. L. Krapivsky, Phys. Rev. E 69, 050901(R) (2004).

[21] D. A. Kessler and N. M. Shnerb, Phys. Rev. E 76, 010901(R) (2007).

[22] L. F. Gordillo, S. A. Marion, A. Martin-Löf, and P. E. Greenwood, Bull. Math. Biol. 70, 589 (2008).

[23] R. van der Hofstad, A. J. E. M. Janssen, and J. S. H. van Leeuwaarden, Adv. Appl. Probab. 42, 1187 (2010).

[24] D. A. Kessler and N. M. Shnerb, arXiv:1201.5306

[25] R. Anderson and R. May, Infectious Diseases: Dynamics and Control (Oxford University Press, Oxford, 1991).
[26] H. W. Hethcote, SIAM Rev. 42, 599 (2000).

[27] G. H. Weiss and M. Dishon, Math. Biosci. 11, 261 (1971).

[28] H. E. Daniels, Biometrika 59, 211 (1972).

[29] C. Lefèvre and P. Picard, Adv. Appl. Prob. 22, 25-48 (1990).

[30] F. Ball and D. Clancy, Adv. Appl. Prob. 25, 721 (1993).

[31] H. Andersson and B. Djehiche, J. Appl. Prob. 35, 662670 (1998).

[32] J. Grasman, Math. Biosci. 152, 13-27 (1998).

[33] R. Antia, R. R. Regoes, J. C. Koella, and C. T. Bergstrom, Nature 426, 658 (2003).

[34] P. Grassberger, Math. Biosci. 63, 157 (1983).

[35] D. Stauffer and A. Aharony, Introduction to Percolation Theory (Taylor \& Francis, Briston PA, 1994).

[36] H. Andersson and B. Djehiche, J. Appl. Prob. 34, 698 (1998).

[37] H. Hinrichsen, Adv. Phys. 49, 815 (2000).

[38] T. Antal, M. Droz, A. Lipowski, and G. Ódor, Phys. Rev. E 64, 036118 (2001).

[39] J. Marro and R. Dickman, Nonequilibrium Phase Transition in Lattice Models (Cambridge, Cambridge University Press, 1999).

[40] J. D. Murray, Mathematical Biology. II. Spatial Models and Biomedical Applications (Springer-Verlag, New York, 2002).

[41] C. P. Warren, L. M. Sander, and I. M. Sokolov, Phys. Rev. E 66, 056105 (2002).

[42] T. Tomé and R. M. Ziff, Phys. Rev. E 82, 051921 (2010).

[43] R. Pastor-Satorras and A. Vespignani, Phys. Rev. Lett. 86, 3200 (2001).

[44] R. M. May and A. L. Lloyd, Phys. Rev. E 64, 066112 (2001).

[45] M. E. J. Newman, Phys. Rev. E 66, 016128 (2002).

[46] T. E. Harris, The Theory of Branching Processes (Dover, New York, 1989).

[47] M. Kimmel and D. Axelrod, Branching Processes in Biology (Springer, New York, 2002).

[48] The distribution describing the populations with no recovered is easy to compute; it turns out to be exponential, $P_{i, 0}=e^{-2 t}\left[\left(1-e^{-2 t}\right) / 2\right]^{i-1}$.

[49] C. J. Ridler-Rowe, J. Appl. Prob. 4, 19 (1967).

[50] N. G. Van Kampen, Stochastic Processes in Physics and Chemistry (North Holland, Amsterdam, 2003).

[51] B. Bollobás, C. Borgs, J. T. Chayes, J. H. Kim, and D. B. Wilson, Rand. Struct. Alg. 18, 201 (2001).

[52] E. Ben-Naim and P. L. Krapivsky, Phys. Rev. E 71, 026129 (2005). 


\section{Appendix A: Inverse Laplace Transforms}

From Eq. (25), the scaling function $\varphi(\eta)$ equals the inverse Laplace transform

$$
\varphi(\eta)=\frac{1}{2 \pi i} \int_{\gamma-i \infty}^{\gamma+i \infty} d b e^{b \eta} \frac{2 \sqrt{b}}{e^{2 \sqrt{b}}+1}
$$

where the integration contour is a line parallel to the imaginary axis in the complex $b$ plane and $\gamma>0$ so that all singularities are to the left of the integration contour. The integrand has simple poles at $b_{k}=-\pi^{2}\left(k+\frac{1}{2}\right)^{2}$, $k=0,1,2, \ldots$ and a branch point at the origin, and we select a branch cut along the negative real axis so that it does not cross the path of integration. Furthermore, we pick a closed Bromwich contour formed by the contour followed by a quarter of a circle of infinitely large radius, followed by the top of the branch cut with infinitesimal half-circles around each pole and then encircling the origin and proceeding similarly along the bottom of the branch cut, followed by a quarter of a circle. By the Cauchy theorem, the integral along this closed contour vanishes. The integrals over the circles vanish and the integrals over the branch cut can be computed to yield

$\varphi(\eta)=2 \pi^{2} \sum_{k=0}^{\infty}\left(k+\frac{1}{2}\right)^{2} e^{-\pi^{2}\left(k+\frac{1}{2}\right)^{2} \eta}-\frac{1}{\pi} \int_{0}^{\infty} d c e^{-c \eta} \sqrt{c}$

where the sum is proportional to the residues of poles at $b_{k}=-\pi^{2}\left(k+\frac{1}{2}\right)^{2}$. Since the integral on the right-hand side equals $(4 \pi)^{-1 / 2} \eta^{-3 / 2}$, we arrive at (26).

The inverse Laplace transform of (34) with respect to $b$ gives the joint scaling function

$$
F(\xi, \eta)=\frac{1}{2 \pi i} \int_{\gamma-i \infty}^{\gamma+i \infty} d b e^{b \eta}\left[\frac{\sqrt{b}}{\sinh \sqrt{b}}\right]^{2} e^{-\xi \sqrt{b} \operatorname{coth} \sqrt{b}} .
$$

To obtain the leading asymptotic behavior in the small- $\eta$ limit, given in (35), we simply evaluate the large- $b$ behavior. In the opposite limit, $\eta \rightarrow \infty$, the asymptotic behavior follows from the singularity of the Laplace transform in the complex $b$ plane that is closest to the origin. The Laplace transform has singularities at $b_{k}=-\pi^{2} k^{2}$, $k=1,2, \ldots$, so we focus on the singularity at $b_{1}=-\pi^{2}$. For a fixed $\xi$, the scaling function appears to decay exponentially, $F(\xi, \eta) \sim e^{-\pi^{2} \eta}$ as $\eta \rightarrow \infty$. To establish the complete asymptotic behavior we recall that a subdominant power-law prefactor, $F(\xi, \eta) \sim \eta^{m} e^{-\pi^{2} \eta}$, implies that the Laplace transform has the algebraic singularity $\left(\pi^{2}+b\right)^{-m-1}$ as $b \rightarrow-\pi^{2}$. In fact, the Laplace transform has the essential singularity (we set $b=-\pi^{2}(1-\epsilon)^{2}$, so that the limit $b \rightarrow-\pi^{2}$ is equivalent to the $\epsilon \rightarrow 0$ limit)

$$
\epsilon^{-2} \exp \left[\left(\epsilon^{-1}-1\right) \xi\right] \text {. }
$$

An essential singularity of the type $e^{\xi / \epsilon}$ corresponds to a subdominant prefactor that is a stretched exponential, $e^{\pi \sqrt{8 \xi \eta}}$. An additional power-law factor times a numerical factor allow to match the singularity (A2). The resulting large- $\eta$ asymptotic behavior is given in (35). 